\title{
Determination of Planck's Constant using Light Emitting Diodes
}

\section{Cliff Orori Mosiori ${ }^{1}$, Duke Ateyh Oeba ${ }^{2}$, Reuben Shikambe ${ }^{2}$}

${ }^{1}$ Technical University of Mombasa

P. 0. Box 90420-80100, Mombasa, Kenya

${ }^{2}$ Kenyatta University

P. 0. Box 43844-00100, Nairobi, Kenya

DOI: $10.22178 /$ pos.27-2

LCC Subject Category: TP155-156, QC450-467, QD450-801

Received 24.07.2017

Accepted 12.09.2017

Published online 20.10.2017

Corresponding Author:

corori@tum.ac.ke

(C) 2017 The Authors. This

article is licensed under a

Creative Commons

Attribution 4.0 License
Abstract. Planck's constant is named after Max Planck, a nineteenth-century physicist who first described it by relating it as $E=h \varpi$ where symbols have their usual meanings. It is a relationship used when comparing a quantum of energy absorbed to that emitted during electron transitions which can be extended to emission by light-emitting diodes. The purpose of this study was to determine Planck's constant using the energy needed to excite free electrons in a light emitting diode. When a light-emitting diode is switched on, electrons recombine with holes within and release energy in the form of photons which can be determined using energy band gaps of the semiconductor composite material used to fabricate the LED. Therefore, LEDs consist of a chip of doped semiconducting layers to create a $p-n$ junction. In LEDs, current flows easily from the $p$-side to the $n$-side but not in the reverse from electrodes with different voltages. When an electron meets a hole, it is inhaled and it falls into lower energy level releasing energy in the form of a photon. Photon emissions take place when electrons return to a lower energy state. Therefore, electrons within a LED crystal are excited to a higher energy state and any radiation emitted depends on the p-n junction direct band gap. Depending on the materials used, LEDs emit radiation with energies corresponding to either near-infrared, visible, or near-ultraviolet light. In reality, a LED is designed to have a small area (approximately less than $1 \mathrm{~mm}^{2}$ ). In this work, an electric current was used to excite electrons and the corresponding energy was measured using a voltmeter. Planck's constant was calculated by substituting the obtained frequency and energy from the voltmeter in the relationship, $E=h \varpi$.

Keywords: LEDs; Bohr frequency; Fermi's Golden Rule; Max Planck; Eigenfunctions; time-independent Schrödinger equation; transition moment; Bloch oscillation.

\section{INTRODUCTION}

Light wavelength can be measured using a spectrometer. Using the relationship, $c=f \lambda$, one can find the frequency emitted by any diode $[4,8,9]$. In this work, we start by considering the quantum mechanics of light absorption between two close states arbitrary taken as state 1 and state 2 , with Eigen-functions with Eigen-values, $\psi_{1}, \psi_{2}$ and with corresponding wave-energies $E_{1}, E_{2}$, respectively. This can be illustrated as shown in Figure 1.

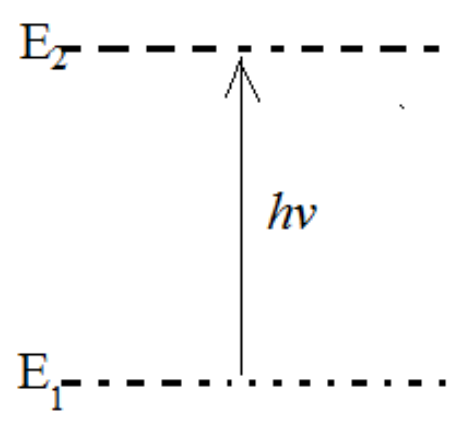

Figure 1 - Energy in close states arbitrary taken as 1 and 2 
According to the Bohr condition $[3,1,5]$, absorption is only "allowed" when the energy of the photon, $h v$, is equal to the energy difference between the two states (state 1 and state 2 ) i. e.:

$$
\Delta E=E_{2}-E_{1}=h v .
$$

Consider an electron in the Bohr orbit of a hydrogen atom and its interaction with electromagnetic radiation especially visible light wave. It's clear that an electron in a particular state at time $t=0$, has a probability to be found in some other state at a later time $t$. In which the transiting energy can be expressed as (2):

$$
\Delta E=E_{2}-E_{1}=h v=h\left(\frac{c}{\lambda}\right)
$$

This expression seems to be easy and direct; however, there are other "rules" that govern such transitions.

Recalling from the quantum mechanics $(Q M)$ postulates that if the functions $\psi_{j}^{0}$ are Eigenfunctions of the time-independent Schrödinger equation, the using the Eigen operators, we obtain the expression for time-independent Schrödinger equation as (3)

$$
\hat{H}^{0} \psi_{j}^{0}=E_{j}^{0} \psi_{j}^{0}
$$

This results into a time-dependent wave-function commonly expressed in the form of (4):

$$
\psi_{j}^{o}=\psi_{j}^{0} e^{-i E_{j} t / \hbar}
$$

In this case, each $\Psi_{j}^{0}$ forms a solution to the time-dependent Schrödinger equation [3], thus easily accepted that (5):

$$
\hat{H}^{0} \Psi^{0}=i \hbar \frac{\partial \Psi^{0}}{\partial t}
$$

Although in this case, each $\Psi_{j}^{0}$ arbitrary represents a stationary state in the system, it's generally accepted that the most general solution of (5) would best be described using a superposition state hence represented by the expression (6):

$$
\Psi^{0}=\sum_{j=0}^{\infty} a_{j} \Psi_{j}^{0}
$$

A superposed expression is not easy to analyze unless it is normalized $[3,7,8]$. So it is necessary that the expression (6) is normalized to give $\sum_{j} a_{j}^{*} a_{j}=1$. Equation 5 implies that each product in the summation $\left(a_{j}^{*} a_{j}\right)$ defines a measure of the probability and therefore, its shows a measure of where the system is likely to be found in a particular state $j$ with energy $E_{j}$. From these assumptions, $\Psi 0$ forms a general function and from the coefficients in the expansion in (6), it becomes easy to determine the probability of finding a particular state $j$ in the system. Light Emitting Diode modeling starts by assuming that an atom has a fixed state near the nucleus. We suppose that at time, $t=0$, the system is described by states labeled by the quantum numbers, $n_{1}, \ell_{1}, m_{\ell_{1}}[3,6,4,5]$. It will be assumed that all other terms in the expansion being zero at time $t=0$ so that (7):

$$
\Psi^{0}=a_{1} \Psi_{1}^{0}, \quad a_{1}=1
$$

Hence, we note that, $\sum_{j} a_{j}^{*} a_{j}=1.0$.

These states start to denote state 1 in which the electron orbits about the nucleus represented schematically as in Figure 2.

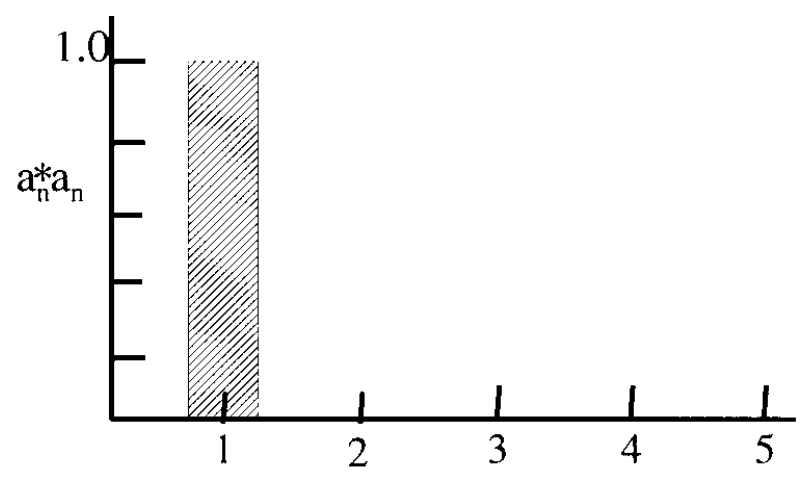

Figure 2 - Electron orbits about the nucleus in state 1

By imagining that a light wave oscillates at some particular frequency, $v$, so that it turns on its perturbation [11], then (8): 


$$
E_{x}=E_{x}^{0} \cos [2 \pi(z / \lambda-v t)] .
$$

At this point, it demands that its probability at some later time, $\mathrm{t}$, will find the system in some other new state 2 . To determine this new state, we find the values of the corresponding coefficients in the expansion expresses in (9):

$$
\Psi=a_{1} \Psi_{1}^{0}+a_{2} \Psi_{2}^{0}+a_{3} \Psi_{3}^{0}+\ldots
$$

These coefficients can be represented schematically as in Figure 3:

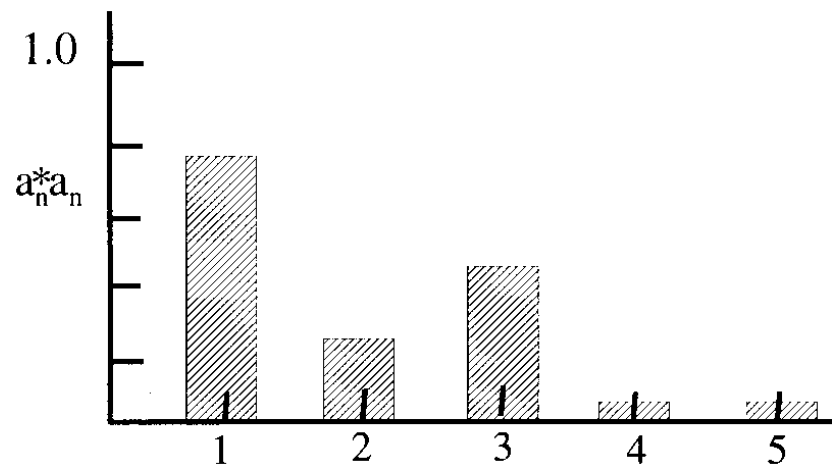

Figure 3 - Corresponding coefficients in the expansion in state 2

For simplicity purposes, we let the system to be analyzed here to have only two states, 1 and 2 where $a_{1}=1.0$ at time $t=0$. We also let the frequency of the light wave obey Bohr frequency condition [3] given as (10):

$$
v=\frac{\left(E_{2}-E_{1}\right)}{h}
$$

Using (9), the wave-function of the system will reduce to (11):

$$
\Psi=a_{1} \Psi_{1}^{0}+a_{2} \Psi_{2}^{0}
$$

Therefore at any time $t$, the time-dependent Schrödinger equation is obeyed hence generalized as (12):

$$
\hat{H} \Psi=\left\{\frac{-\hbar^{2}}{2 m} \nabla^{2}+V\right\} \Psi=i \hbar \frac{\partial \Psi}{\partial t}
$$

Form (12) the potential energy function, $V$, include Coulomb potential $\left(-\mathrm{Ze}^{2} / \mathrm{r}\right)$, which produces a perturbation interaction on the electric field, $E_{0}^{o}$ due to the light wave with an electric dipole oriented in $\boldsymbol{x}$-direction expressed [4] as (13):

$$
\hat{H}^{\prime}=-e x E_{x}^{0} \cos [2 \pi(z / \lambda-v t)]
$$

If (12) and (13) are substituted into (11), and then simplify them further using (8), we obtain the time dependence of the coefficients $a_{1}$ and $a_{2}$ explicitly as (14):

$$
-e x E_{x}^{0} \cos [2 \pi(z / \lambda-v t)]\left[a_{1}(t) \Psi_{1}^{0}+a_{2}(t) \Psi_{2}^{0}\right]=i \hbar\left(\Psi_{1}^{0} \frac{d a_{1}(t)}{d t}+\Psi_{2}^{0} \frac{d a_{2}(t)}{d t}\right)
$$

Applying the orthogonality of the unperturbed functions [2], $\Psi_{j}^{0}$, and by multiplying both sides of (14) by an Eigen-function $\Psi_{2}^{*}$ and finally integrating the resulting expression, we obtain (15):

$$
i \lambda \frac{d a_{2}(t)}{d t}=-e E_{x}^{0} a_{1}(t) \cos (2 \pi v t) \int \Psi_{2}^{*} \hat{x} \Psi_{1} d \tau
$$

In (15) the quantity $\frac{d a_{2}(t)}{d t}$ represents the rate of transitions from state 1 to state 2 and therefore solving for $a_{2}(t)$ within very short times spans, $(t) \sim 1$, and $\Psi_{2}^{*}, \Psi_{1} \rightarrow \psi_{2}^{*}, \psi_{1}$, will yield (16):

$$
a_{2}(t) \sim-\frac{e E_{x}^{0} t}{i \hbar} \int \psi_{2}^{*} \hat{x} \psi_{1} d \tau
$$


Such a modelled (16) reduces the probability of finding the atom in the state 2 at time $t$ into the Fermi's Golden Rule [6] and can be expressed as (17):

$$
a_{2}^{*} a_{2} \sim \frac{e^{2}\left(E_{x}^{0}\right)^{2} t^{2}}{\hbar^{2}}\left|\int \psi_{2}^{*} \hat{x} \psi_{1} d \tau\right|^{2}
$$

Using Fermi's Golden Rule in (17), one can to derive the selection rules for any electric dipole transition resulting into a transition moment [5] expressed as (18):

$$
\int \psi_{2}^{*} e \hat{x} \psi_{1} d \tau \equiv \mu_{12}
$$

In (18), the equivalent value, $\mu_{12}$ must be nonzero if the transition is to allowed; otherwise, its for-

$$
\left\{\begin{array}{l}
\mu_{x} \\
\mu_{y} \\
\mu_{z}
\end{array}\right\}=\int_{0}^{\infty} R_{n \ell} \mu R_{n^{\prime \prime} \ell^{\prime \prime}} r^{2} d r \times \int_{0}^{\pi} \Theta_{\ell m}\left\{\begin{array}{l}
\sin \theta \\
\sin \theta \\
\cos \theta
\end{array}\right\} \Theta_{\ell^{\prime \prime} m^{\prime \prime}} \sin \theta d \theta \times \int_{0}^{2 \pi} \Phi_{\mathrm{m}}^{*}\left\{\begin{array}{c}
\cos \psi \\
\sin \psi \\
1
\end{array}\right\} \Phi_{\mathrm{m}} d \phi
$$

bidden. Therefore, the dipole moment operator for two particles which have charges $\pm e$ is $\mu(r)=e r$. Its components will be expressed as (19):

$$
\begin{aligned}
& \mu_{x}=\mu(r) \sin \theta \cos \phi \\
& \mu_{y}=\mu(r) \sin \theta \cos \phi \\
& \mu_{z}=\mu(r) \cos \phi
\end{aligned}
$$

Finally, to determine the selection rules for electronic transitions in the hydrogen atom [11], it is required that (20) be evaluated to obtain the selection rules for $n, \ell_{\mathrm{m}}$ and $\mathrm{m}_{\ell}$ which are $\Delta n=$ anything $, \Delta \ell= \pm 1, \Delta m_{\ell}=0, \pm 1$ :

\section{METHODOLOGY}

\section{Materials}

The following were used to ensure success of this investigation: Computer; Vernier interface; Vernier spectrometer with USB cable; fiber optic cable for the spectrometer; voltage sensor LED exciter box; collection of LED's; labels or masking tape; and an alternative - Use a Spectroscope to determine wavelength among others.

\section{Procedure}

A LED was chosen and inserted into the exciter box to ensure that it completes the circuit with the longer leg connected to the red wire and to the internal resistor in the correct polarity. A fiber optic cable was attached to the spectrometer and connected to a computer using a USB cable enabled to run Logger Pro software. The intensity was adjusted so as not to be too low or too

\begin{tabular}{|c|c|c|}
\hline $\begin{array}{l}\text { Wavelength } \\
\text { (nm) }\end{array}$ & $\begin{array}{c}\text { Potential } \\
\text { (volts) }\end{array}$ & $\begin{array}{c}\text { Planck's Constant } \\
(\mathrm{J} \cong \mathrm{s})\end{array}$ \\
\hline 564 & 2.00 & $6.02 \times 10^{-34}$ \\
\hline 591 & 1.92 & $6.07 \times 10^{-34}$ \\
\hline 594 & 1.96 & $6.20 \times 10^{-34}$ \\
\hline 610 & 1.87 & $6.08 \times 10^{-34}$ \\
\hline 618 & 1.88 & $6.19 \times 10^{-34}$ \\
\hline 625 & 1.84 & $6.12 \times 10^{-34}$ \\
\hline 627 & 1.83 & $6.12 \times 10^{-34}$ \\
\hline 635 & 1.83 & $6.20 \times 10^{-34}$ \\
\hline 646 & 1.66 & $5.73 \times 10^{-34}$ \\
\hline 685 & 1.97 & $7.21 \times 10^{-34}$ \\
\hline
\end{tabular}
high using the distance from the tip of the cable to the LED. Both the peak and maximum wavelength of the LED and the color of the LED's light were recorded in Table 1.
Table 1 - Experimental calculated Planck's constant

This was repeated for each of the available LED's. The spectrometer was then unplugged from the computer and instead a voltage sensor into a Vernier interface. Finally, the mode of data collection was changed from "Time Based "to "Events with Entry" and then run. Thereafter, "Connect Points" was disabled on the Graph Options menu and then run using a computer.

Lastly, the wavelength of unknown LED could be determined if proposed to be determined. 


\section{RESULTS AND DISCUSSION}

The purpose of this study was to determine Planck's constant using the energy needed to excite free electrons in a light emitting diode. In this work, an electric current was used to excite electrons and the corresponding energy was measured using a voltmeter as it was emitted. Planck's constant was then calculated by substituting the obtained frequency and energy from the voltmeter in the relationship, $E=h \varpi[6]$. A LED' emitting red, orange, yellow and yellow-green and green color was also tested but the results are available elsewhere. The results were tabulated in Table 1.

Based on these findings of Planck's constant, Zener tunneling was proposed to be analyzed based on this semi classical model. In Zener diodes, an electron can jump to new band energy as explained by classical models. It was further proposed that this work can be extended to explain any change and causes that can cause a ruin on the Bloch oscillation since according to Marder's [1] the tunneling amplitude based on the WKB approximation, the amplitude can be determined from (21):

$$
T \approx \exp \left[-\frac{\varepsilon_{g}}{e E} \sqrt{\frac{2 m \varepsilon_{g}}{\hbar^{2}}}\right] .
$$

Since (21) is an exponential function with 1 /e as the amplitude, the 'e-fold' point [8] can be reduced to (22):

$$
\frac{\varepsilon_{g}}{e E} \sqrt{\frac{2 m \varepsilon_{g}}{\hbar^{2}}}=1
$$

Thus, the size of atomic fields [12] may equally be explained and be determined using the Planck's constant as implied in (23) through Zener tunneling as:

$$
E=\frac{\varepsilon_{g}}{e} \sqrt{\frac{2 m \varepsilon_{g}}{\hbar^{2}}} \approx 1.5 \times 10^{10} \mathrm{~V} / \mathrm{m}
$$

Hence, the electric field can be found similarly using an electron orbiting at the Bohr radius [11] around a proton or nucleus.

\section{CONCLUSION}

Planck's constant was determined by measuring the energy emitted by a selected number of light emitting diodes. A spectrometer was used to measure the wavelength of the light emitted by each LED. A measure of voltage drop across each light emitting diode was used to Planck's constant by relating it to the energy absorbed/emitted by the diodes through calculating it from the wavelength and voltage. It was found to be approximately similar to the theoretical value whereby it was concluded that the proposed method can be used to determine the wavelength of light emitted by unknown LED.

\section{REFERENCES}

1. Altschul, B., Bailey, Q. G., Blanchet, L., Bongs, K., Bouyer, P., Cacciapuoti, L., ... Wolf, P. (2015). Quantum tests of the Einstein Equivalence Principle with the STE-QUEST space mission. Advances in Space Research, 55(1), 501-524. doi: 10.1016/j.asr.2014.07.014

2. Asano, M., Basieva, I., Khrennikov, A., Ohya, M., Tanaka, Y., \& Yamato, I. (2015). Quantum Information Biology: From Information Interpretation of Quantum Mechanics to Applications in Molecular Biology and Cognitive Psychology. Foundations of Physics, 45(10), 1362-1378. doi: 10.1007/s10701-015-9929-y

3. Ballentine, L. E. (2008). Quantum mechanics: a modern development (2nd ed.). Singapore: World Scientific.

4. De Ronde, C. (2015). Modality, Potentiality, and Contradiction in Quantum Mechanics. New Directions in Paraconsistent Logic, 249-265. doi: 10.1007/978-81-322-2719-9_11

5. Dragoman, D. (2005). Phase Space Formulation of Quantum Mechanics. Insight into the Measurement Problem. Physica Scripta, 72(4), 290-296. doi:

10.1238/physica.regular.072a00290 
6. Henson, J. (2015). Bounding Quantum Contextuality with Lack of Third-Order Interference. Physical Review Letters, 114(22). doi: 10.1103/physrevlett.114.220403

7. Leggett, A. J. (2008). Realism and the physical world. Reports on Progress in Physics, 71(2), 022001. doi: 10.1088/0034-4885/71/2/022001

8. Peña, L. (2016). EMERGING QUANTUM: the physics behind quantum mechanics. N. d.: Springer.

9. Reinisch, G. (1994). Nonlinear quantum mechanics. Physica A: Statistical Mechanics and Its Applications, 206(1-2), 229-252. doi: 10.1016/0378-4371(94)90128-7

10. Svensson, B. E. Y. (2014). On the interpretation of quantum mechanical weak values. Physica Scripta, T163, 014025. doi: 10.1088/0031-8949/2014/t163/014025

11. Weinberg, S. (1989). Testing quantum mechanics. Annals of Physics, 194(2), 336-386. doi: 10.1016/0003-4916(89)90276-5

12. Znojil, M. (2004). Relativistic supersymmetric quantum mechanics based on Klein-Gordon equation. Journal of Physics A: Mathematical and General, 37(40), 9557-9571. doi: 10.1088/0305-4470/37/40/016 